\title{
Explosive Percolation is Continuous, but with Unusual Finite Size Behavior
}

\author{
Peter Grassberger, ${ }^{1,2}$ Claire Christensen, ${ }^{2}$ Golnoosh Bizhani, ${ }^{2}$ Seung-Woo Son, ${ }^{2}$ and Maya Paczuski ${ }^{2}$ \\ ${ }^{1}$ FZ Jülich, D-52425 Jülich, Germany \\ ${ }^{2}$ Complexity Science Group, University of Calgary, Calgary T2N 1N4, Canada
}

(Received 23 March 2011; published 31 May 2011)

\begin{abstract}
We study four Achlioptas-type processes with "explosive" percolation transitions. All transitions are clearly continuous, but their finite size scaling functions are not entirely holomorphic. The distributions of the order parameter, i.e., the relative size $s_{\max } / N$ of the largest cluster, are double humped. But-in contrast to first-order phase transitions-the distance between the two peaks decreases with system size $N$ as $N^{-\eta}$ with $\eta>0$. We find different positive values of $\beta$ (defined via $\left\langle s_{\max } / N\right\rangle \sim\left(p-p_{c}\right)^{\beta}$ for infinite systems) for each model, showing that they are all in different universality classes. In contrast, the exponent $\Theta$ (defined such that observables are homogeneous functions of $\left.\left(p-p_{c}\right) N^{\Theta}\right)$ is close to-or even equal to- $1 / 2$ for all models.
\end{abstract}

DOI: 10.1103/PhysRevLett.106.225701

PACS numbers: 64.60.ah, 05.40.-a, 05.70.Jk, 89.75.Da

Percolation is a pervasive concept in statistical physics and probability theory and has been studied in extenso in the past. It came thus as a surprise to many when Achlioptas et al. [1] claimed that a seemingly mild modification of standard percolation models leads to a discontinuous phase transition-named "explosive percolation" (EP) by them -in contrast to the continuous phase transition seen in ordinary percolation. Following [1] there appeared a flood of papers [2-20] studying various aspects and generalizations of EP. In all cases, with one exception [20], the authors agreed that the transition is discontinuous: the "order parameter", defined as the fraction of vertices or sites in the largest cluster, makes a discrete jump at the percolation transition. Here we join the dissenting minority and add further strong evidence that the EP transition is continuous in all models, but with unusual finite size behavior (recently, continuity of EP was also shown in [21]).

From the physical point of view, the model seems somewhat unnatural. To establish a new link, a set of "candidate" links is chosen at random, only one of which is really established. This is done such that the formation of large clusters is delayed, as compared to random (bond) percolation. Since the nonchosen candidate links will in general connect distant nodes in the final network, this implies nonlocal control [22]. Also, notwithstanding [8], no realistic applications have been proposed. It is well known that including long-range interactions can alter the universality class, or drive a transition first order. Thus it is not so surprising that a percolation model with global control can show different behavior [23].

Usually, e.g., in thermal equilibrium systems, discontinuous phase transitions are identified with "first-order" transitions, while continuous transitions are called "second order." This notation is also often applied to percolative transitions. But EP lacks most attributes-except possibly for the discontinuous order parameter jump-considered essential for first-order transitions. None of these other attributes (cooperativity, phase coexistence, and nucleation) is observed in Achlioptas type processes, although they are observed in other percolation-type transitions [24]. Thus EP should never have been viewed as a first-order transition, and it is gratifying that it is also not discontinuous.

Apart from the behavior of the average value $\langle m\rangle$ of the order parameter $m$, phase transitions can also be characterized by the distribution $P_{p, N}(m)$ of $m$ in finite systems, where $p$ is the control parameter and $N$ measures the system size. For infinite $N,\langle m\rangle$ jumps at $p=p_{c}$ if the transition is discontinuous, while it varies continuously with a power law singularity $\langle m\rangle \sim\left(p-p_{c}\right)^{\beta}$ for a continuous transition. The distribution $P_{p=p_{c}, N}(m)$ at criticality scales, for continuous transitions, as [25]

$$
P_{p=p_{c}, N}(m) \sim N^{\eta} f\left(m N^{\eta}\right),
$$

where $\eta=\beta /(d \nu)$ for standard thermal second order phase transitions. The universal function $f(z)$ might be double humped, as in the Ising model [25]. But then, as $N \rightarrow \infty$, the dip between the humps usually does not deepen and the horizontal distance between them shrinks to zero so that $P_{p=p_{c}, N}(m)$ becomes single humped.

Equation (1) is directly related to the finite size scaling (FSS) of $\langle m\rangle$ [26],

$$
\langle m\rangle \sim\left(p-p_{c}\right)^{\beta} g\left[\left(p-p_{c}\right) N^{\Theta}\right],
$$

where the universal scaling function $g(z)$ is analytic at all finite $z$, reflecting the fact that the critical point was the only singularity of the partition function, before it was regularized by Eq. (2). Notice that the usual FSS ansatz [26] involves the linear system size $L$ instead of $N$ with $\Theta=1 /(d \nu)$, where $d$ is the dimension and $\nu$ is the correlation length exponent.

In typical first-order transitions, in contrast, $P_{p=p_{c}, N}(m)$ is double humped with a deepening valley between the two 
peaks. The distance between the peaks tends to a positive constant which is equal to the jump in $\langle m\rangle$. The depth of the valley between the peaks reflects the fact that values of $m$ between the peaks correspond to systems with two coexisting phases and an interface between them that costs energy and is disfavored. As a consequence, systems with first-order transitions typically do not show FSS (unless the interface energy does not increase with system size [27]).

In percolation, usually the relative size of the largest cluster, $m \equiv s_{\max } / N$, is taken as an order parameter. Here, $N$ is the number of nodes, and $s_{\max } / N \rightarrow 0$ for $p<p_{c}$ and $N \rightarrow \infty$. In $[6,16]$ it was observed that $P_{p=p_{c}, N}(m)$ is strongly double peaked in EP transitions. In [16] this was also backed by careful measurements of the depth of the valley between the peaks, which indeed lowered with increasing $N$. This was taken as a clear indication for the transition being first order and for phase coexistence. Notice that the latter is not justified since $s_{\max } / N$ is, in contrast to the local order parameters in thermal systems, a global quantity and cannot be used to characterize any part of a large system. Rather, the structure of $P_{p=p_{c}, N}(m)$ in EP reflects the suddenness of the transition, combined with a scatter of the precise $p$ values where individual systems acquire giant clusters. At $p$ values where both peaks have the same height, it is much more likely to find either no giant cluster or a fully developed one, than to find a halfgrown giant cluster. Hence, the two peaks are more reminiscent of systems without self-averaging [28] than of phase coexistence.

While the two peaks prove the suddenness of the transition that was claimed as a hallmark of EP, they do not yet prove that EP is discontinuous. For that, one must also show that the distance between the peaks does not vanish for $N \rightarrow \infty$. In order to check this, we have made extensive simulations of four models: The original product rule of [1], denoted in the following as "PR"; The product rule on 2D square lattices $[3,4]$ with helical boundary conditions ("2D"); The "adjacent edge" rule [7] ("AE"); And the rule of [20] ("CDGM"). For details on the simulations, see the supplemental material (SM) [29].

Distributions $P_{p, N}(m)$ for these models are shown in Fig. 1. In all cases $p$ was chosen such that both peaks have equal height (set arbitrarily to 1). The extrapolations of these values for $N \rightarrow \infty$ are given in Table I. They agree within errors with the critical $p_{c}$ values quoted in the literature. We see that in each case the valley between the peaks deepens with increasing $N$ [16], but at the same time both peaks shift to the left. Among the three off-lattice models, the AE model (the least nonlocal) shows the fastest peak shifting and slowest valley deepening, while the opposite is true for the CDGM model. In all cases this shift is compatible with power laws

$$
m_{ \pm} \sim N^{-\eta_{ \pm}}
$$

where $m_{+}\left(m_{-}\right)$is the position of the right (left) peak at the critical point. In all cases $0<\eta_{+}<\eta_{-}$(see Table I), i.e.,

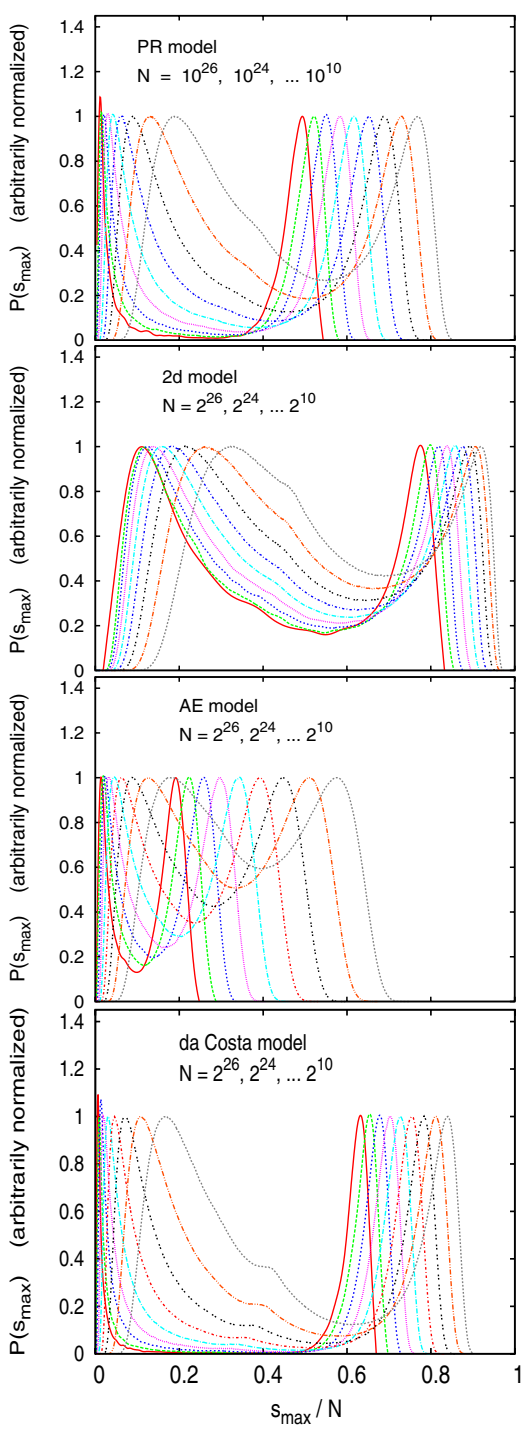

FIG. 1 (color online). Distributions of the order parameter $s_{\text {max }} / N$ for four EP models. They are shown at the effective critical point, defined such that both peaks have the same height. Normalization is such that their height is 1. For the largest systems, curves were approximated by cubic splines to make them smooth.

the right peak moves slower than the left one. Therefore the distance between the peaks increases for small $N$, but finally decreases $\sim N^{-\eta_{+}}$. When $p$ is chosen such that the two peaks have equal area, the distance between them is asymptotically proportional to the maximum of the variance of $m$. Since the peak positions change very little when passing from equal heights to equal masses [16], the variances first increase with $N$ (in agreement with [4]), but ultimately decreases.

Indeed, basing Eq. (3) on peak positions when both peaks have equal areas would seem more natural than basing it on equal peak heights. It was not done in Figs. 1 and 2 for purely technical reasons-peak areas are difficult to define for shallow minima, and the shifts in position would be visually less evident in view of the 
TABLE I. Critical points and critical exponents for the four models. The $\Theta_{i}$ are different estimates of the exponent $\Theta: \Theta_{1}$ is obtained from the scaling relation $\Theta=\eta_{+} / \beta, \Theta_{2}$ is obtained from a data collapse in the slightly supercritical region where $\langle m\rangle \approx m_{+}$, and $\Theta_{\text {conj }}$ is the conjectured exact value. For the CDGM model, $p_{c}$ is taken from [20]. For the other models it is obtained from plots analogous to the inset in Fig. 4.

\begin{tabular}{lcccc}
\hline \hline & PR & $2 \mathrm{~d}$ & AE & CDGM \\
\hline$p_{c}$ & $0.888449(2)$ & $0.526562(3)$ & $0.797013(3)$ & 0.923207508 \\
$\eta_{+}$ & $0.0402(15)$ & $0.018(2)$ & $0.103(2)$ & $0.0255(8)$ \\
$\eta_{-}$ & $0.270(7)$ & $0.078(7)$ & $0.228(5)$ & $0.300(5)$ \\
$\beta$ & $0.0861(5)$ & $0.040(2)$ & $0.214(2)$ & $0.0557(5)$ \\
$\Theta_{1}$ & $0.47(2)$ & $0.45(6)$ & $0.48(1)$ & $0.46(2)$ \\
$\Theta_{2}$ & $0.52(1)$ & $0.47(3)$ & $0.51(1)$ & $0.53(1)$ \\
$\Theta_{\text {conj }}$ & $1 / 2$ & - & $1 / 2$ & $1 / 2$ \\
$\eta_{0}$ & $0.0567(9)$ & $0.0612(8)$ & $0.1113(8)$ & $0.0356(8)$ \\
\hline \hline
\end{tabular}

vastly different heights. The resulting values of $\eta_{ \pm}$would be slightly larger (by 5\% to 10\%) than those given in Table I. This would eliminate the small differences between $\Theta_{1}$ and $\Theta_{2}$ discussed below, but it would not otherwise affect our conclusions.

As shown in Fig. 2 for the CDGM model, not only the positions of the peaks scale, but also their widths. This indicates that the asymptotic scenario is two well separated peaks with $N$-independent shapes whose widths are proportional to their positions. If we switch from defining $p_{c}$ by equal peak heights to equal peak areas and allow weak convergence for $N \rightarrow \infty$ (in contrast to the usual assumption of pointwise convergence; see the SM [29]) the full distributions at $p_{c}(N)$ then show asymptotic scaling
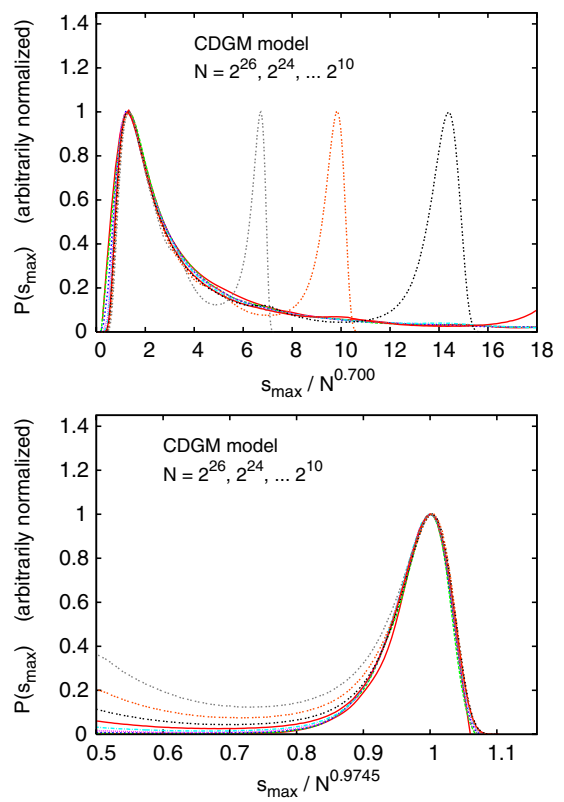

FIG. 2 (color online). Data collapses for the two peaks in the order parameter distribution for the CDGM model. Colors and line styles are the same as in Fig. 1.

$$
P_{p_{c}(N), N}(m) \sim N^{\eta_{+}} f\left(m N^{\eta_{+}}\right)
$$

with the scaling function $f(x)$ consisting of a finite width right hand peak and a $\delta$ peak at $x=0$.

For $p$ strictly larger than $p_{c}(N)$, only the right hand peak dominates the average $\langle m\rangle$. We then expect only small finite size scaling corrections to its asymptotic values, i.e., we expect the curves $\langle m\rangle_{p, N}$ for different $N$ to coincide for $p>p_{c}(N)$ on a common curve $\langle m\rangle_{p}$. Since the scenario in this regime is not much different from other critical phenomena this should be a power law $\langle m\rangle \sim\left(p-p_{c}\right)^{\beta}$ that holds in the range $m_{+}<\langle m\rangle \ll 1$. Measured values of $\beta$ are given in Table I. For the CDGM model the agreement with [20] is perfect. Assuming Eq. (2), it follows that $\Theta=$ $\eta_{+} / \beta$. Values of $\Theta$ obtained from this, denoted as $\Theta_{1}$, are slightly smaller than $1 / 2$ for all models (see Table I).

Deviations from this common power law are expected to set in when $\langle m\rangle$ decreases below $m_{+}$. The data for the CDGM model are shown in Figs. 3. For all $p>p_{c}$ (except for very small values of $\left.z=\left(p-p_{c}\right) N^{\Theta}\right)$, these deviations are fully described by the FSS ansatz in Eq. (2). In Figs. 3 we chose $\Theta$ so that the collapse is best at $\langle m\rangle \approx m_{+}$, resulting in the value $\Theta_{2}$ quoted in Table I. For the other models the data collapse is similarly good, except for the 2D model where it is worse (see SM). For all models, $\Theta_{2}$ is slightly larger than $\Theta_{1}$.

The fact that $f(z)$ in Eq. (4) contains a $\delta$ peak at its leftmost extremity $z=0$ implies that $g(z)$ in Eq. (2) must vanish for all $z$ below some value $z_{0} \leq 0$, which in turn means that $g(z)$ must have a singularity at $z_{0}$. Indeed, Fig. 4 shows that the values of $g(z)$ for $z<-1$ approach 0 very fast with increasing $N$, implying $-1<z_{0} \leq 0$ (the latter is also true for the other models). We cannot exclude the possibility the curves in Fig. 4 approach a pure power law $a z^{\beta}$ (dashed red line) in the limit $N \rightarrow \infty$.

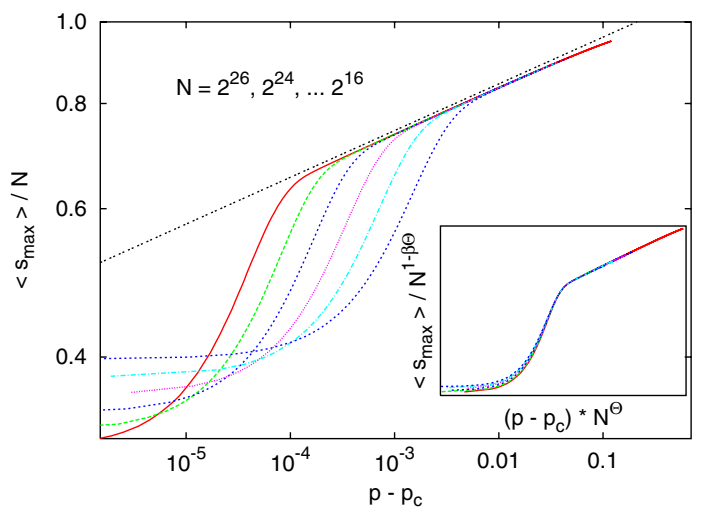

FIG. 3 (color online). Log-log plot of the average order parameter for the CGDM model versus $p-p_{c}$, for six different values of $N$. One sees clearly a common part with slope $\beta$ (indicated also by the straight line), from which curves for different $N$ deviate later and later, as $N$ increases. The inset shows the collapse of these data as predicted by Eq. (2). While $\Theta$ is fitted, both $\beta$ and $p_{c}$ are taken from [20]. 


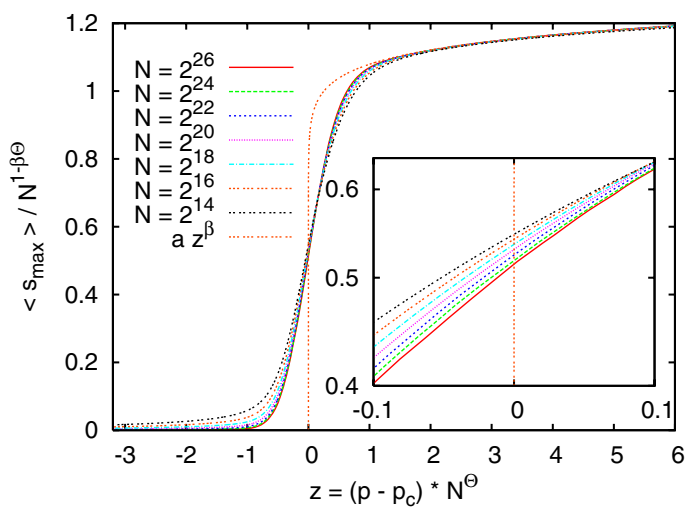

FIG. 4 (color online). Doubly linear plot of the same data shown in Fig. 3, but extended to values $p<p_{c}$. Here we used $\Theta=1 / 2$, which gives worse data collapse for $p>p_{c}$, but vastly more systematic behavior for $p<p_{c}$. The inset shows a blowup of the region around $p=p_{c}$, with logarithmic $y$ axis. The decrease of the curves at $z=0$ with $N$ suggests that $z_{0}=0$, and that a new power law holds for $p=p_{c}$.

The blowup of the region around $z=0$ shown in the inset in Fig. 4 hints at a power law $\left.\langle m\rangle\right|_{p=p_{c}} \sim N^{-\eta_{0}}$ with $\eta_{0}=0.0356(8)>\eta_{+}$(see also SM). The same is qualitatively true for the other models, where always $\eta_{0}>\eta_{+}$ (see Table I). We find therefore that $z=0$ is no longer in the realm of uniform pointwise convergence to the FSS ansatz, and hence that $z_{0}=0$. We finally mention that we used $\Theta=0.5$ in Fig. 4, a value in between $\Theta_{1}$ and $\Theta_{2}$, as it gives the simplest behavior for $z<0$. The same is true for the other off-lattice models (but not for the 2D model, see [29]), when we conjecture that $\Theta=0.5$ for them.

The singularity of $g(z)$ at $z=0$ implies also that one cannot expect the effective critical points to scale as $p_{c}(N)-p \sim N^{-\Theta}$. Results obtained for the CDGM model, with $p_{c}(N)$ defined via equal peak masses, are shown in the SM [29]. They indicate that $p_{c}(N)-p \sim$ $N^{-\delta}$ with $\delta=0.9(1)>\Theta$. The agreement with the prediction $\delta=0.818(1)$ of [20]—based on "standard scaling relations" - seems fortitious.

In this Letter we do not present a detailed theory for the convergence to $g(z)$ for $z \leq 0$, in particular, we do not explain how $\eta_{0}$ and $\delta$ are related to $\eta_{-}$. Such a theory is presumably formulated more easily by using either $\left\langle\log s_{\max }\right\rangle$ or $\left\langle 1 / s_{\max }\right\rangle$ as an order parameter. But this would be beyond the scope of the present Letter.

In summary, we have shown that four models of explosive percolation, including the original product rule of Achlioptas et al. [1], have continuous transitions. Each is in a different universality class, but all of them show unusual finite size behavior with a nonanalytic scaling function. They all show double-peaked order parameter distributions with the sharpness of the peaks increasing with system size, and different scaling laws for the width of the scaling region $\left(\sim N^{-\Theta}\right)$ and for the shift of the effective $p_{c}(N)$. This scenario is not found for other recent models $[9,17,30]$ that do indeed show discontinuous transitions. It could be that the features found in the present Letter arise from the specific nonlocality of the Achlioptas process, and that this is why it was not seen previously in other critical phenomena.

We are indebted to Bob Ziff and Liang Tian for most useful correspondence.

[1] D. Achlioptas, R. M. D'Souza, and J. Spencer, Science 323, 1453 (2009).

[2] E. J. Friedman and A. S. Landsberg, Phys. Rev. Lett. 103, 255701 (2009).

[3] R. M. Ziff, Phys. Rev. Lett. 103, 045701 (2009).

[4] R. M. Ziff, Phys. Rev. E 82, 051105 (2010).

[5] F. Radicchi and S. Fortunato, Phys. Rev. Lett. 103, 168701 (2009).

[6] F. Radicchi and S. Fortunato, Phys. Rev. E 81, 036110 (2010).

[7] R. M. D'Souza and M. Mitzenmacher, Phys. Rev. Lett. 104, 195702 (2010).

[8] H. D. Rozenfeld, L. K. Gallos, and H. A. Makse, Eur. Phys. J. B 75, 305 (2010).

[9] S.S. Manna and A. Chatterjee, Physica (Amsterdam) 390A, 177 (2011).

[10] A. A. Moreira et al., Phys. Rev. E 81, 040101 (2010).

[11] Y. S. Cho, B. Kahng, and D. Kim, Phys. Rev. E 81, 030103 (2010).

[12] Y. S. Cho et al., Phys. Rev. Lett. 103, 135702 (2009).

[13] Y. S. Cho et al., Phys. Rev. E 82, 042102 (2010).

[14] N. A. M. Araújo and H. J. Herrmann, Phys. Rev. Lett. 105, 035701 (2010).

[15] U. Basu et al., arXiv:1008.4293.

[16] L. Tian and D.-N. Shi, arXiv:1010.5990.

[17] W. Chen and R. M. D’Souza, Phys. Rev. Lett. 106, 115701 (2011).

[18] J. Nagler, A. Levina, and M. Timme, Nature Phys. 7, 265 (2011).

[19] H. Hooyberghs and B. Van Schaeybroeck, Phys. Rev. E 83, 032101 (2011).

[20] R. A. da Costa et al., Phys. Rev. Lett. 105, 255701 (2010).

[21] O. Riordan and L. Warnke, arXiv:1102.5306.

[22] In contrast to claims made by the authors, the model in [7] is also nonlocal by any standard physics definition.

[23] C. Christensen et al., arXiv:1012.1070.

[24] H.-K. Janssen, M. Müller, and O. Stenull, Phys. Rev. E 70, 026114 (2004).

[25] A. D. Bruce and N. B. Wilding, Phys. Rev. Lett. 68, 193 (1992).

[26] K. Binder and D. W. Heermann, Monte Carlo Simulations in Statistical Physics (Springer, Heidelberg, 2010), 5th ed.

[27] M. S. Causo, B. Coluzzi, and P. Grassberger, Phys. Rev. E 62, 3958 (2000).

[28] S. Wiseman and E. Domany, Phys. Rev. Lett. 81, 22 (1998).

[29] See supplemental material at http://link.aps.org/ supplemental/10.1103/PhysRevLett.106.225701.

[30] S. V. Buldyrev et al., Nature (London) 464, 1025 (2010). 